\title{
Reflujo vesicoureteral complejo. Revisión de nuestra serie
}

\author{
E. Argüelles Salido*, F. García Merino, A. Millán López, M. Fernández Hurtado, \\ J. Borrero Fernández
}

*Servicio de Urología. Servicio de Cirugía Pediátrica. Unidad de Urología Infantil. Hospital Universitario Virgen del Rocío. Sevilla

Actas Urol Esp 2005; 29 (7): 685-692

\section{RESUMEN \\ REFLUJO VESICOURETERAL COMPLEJO. REVISIÓN DE NUESTRA SERIE}

Objetivos: Analizar la proporción de reflujos secundarios o complejos en el total de reflujos vesicoureterales tratados endoscópicamente en nuestro hospital. Determinar el éxito conseguido y su relación con el grado de reflujo presentado.

Material y método: Presentamos nuestra experiencia entre 1992 y 2003 con tres tipos de sustancias (politetrafluoroetileno, polidimetilsiloxane y dextranomero-copolímero de ácido hialurónico). Tratamos endoscópicamente con la inyección de estas sustancias a 74 pacientes con reflujo vesicoureteral complejo. Todos ellos se siguieron sistemáticamente mediante cistografía a los 3 y 9 meses tras la inyección. Definimos éxito del tratamiento como aquellos reflujos grado 0 ó I.

Resultados: El reflujo fue solucionado endoscópicamente en el 86,25\% de las ocasiones en el primer intento, $93,75 \%$ tras el segundo y $96,25 \%$ tras el tercero. El éxito según el grado de reflujo fue $88,9 \%$ para los de grado II, $83,3 \%$ para los de grado III y $100 \%$ para los de IV.

Conclusiones: Concluimos que la inyección subureteral de distintas sustancias (Teflon ${ }^{\circledR}$, Macroplastique ${ }^{\circledR}$ o Deflux ${ }^{\circledR}$ ) constituye una alternativa útil de tratamiento en la mayoría de reflujos vesicoureterales complejos. Opinamos que debe considerarse como el primer escalón del tratamiento.

Palabras clave: Reflujo vesicoureteral. Tratamiento endoscópico. Complejo. Vejiga.

\section{ABSTRACT}

\section{COMPLEX VESICOURETERAL REFLUX. OUR EXPERIENCE}

Objective: To analize the proportion of complex reflux in the whole amount of patients treated endoscopically of vesicoureteral reflux in our hospital. To determine the endoscopic treatment success in complex reflux, and the influence of reflux grade in it.

Material and methods: We present our experience between 1992 and 2003 with three kinds of substances (polytetrafluoroethylene, polydimethylsiloxane and dextranomer-hyaluronic acid copolymer). We treated complex reflux in 74 patients with endoscopic injection. All patients were scheduled to have voiding cystourethrogram 3 and 9 moths after injection. A positive response was defined as grade 0 or I reflux.

Results: Reflux was solved using the endoscopic procedure in $86,25 \%$ after first injection, 93,75\% after second and $96,25 \%$ after third. The corresponding results for reflux grade II, III and IV were $88.9 \%, 83.3 \%$ and $100 \%$.

Conclusions: We conclude that subureteral injection of different sustances (Teflon ${ }^{\circledR}$, Macroplastique ${ }^{\circledR}$ or Deflux ${ }^{\circledR}$ ) is a useful treatment for most cases of vesicoureteral reflux. We propose it as first step of treatment.

Keywords: Vesicoureteral reflux. Endoscopic treatment. Complex. Bladder. 
$\mathrm{E}$ 1 reflujo vesicoureteral (RVU) es una patología frecuente en la edad pediátrica, con una incidencia de un 0,5-1\% aproximadamente y sin diferencia entre sexos ${ }^{1}$.

Se observa en el $50 \%$ de los niños que presentan infecciones del aparato urinario. La insuficiencia renal debido a nefropatía por reflujo supone un $25 \%$ de los niños en diálisis, que se estiman en un 0,5 por millón de habitantes. Estas cifras delatan la importancia del proble$\mathrm{ma}^{2}$.

El tratamiento del RVU viene supeditado principalmente al grado del reflujo. En términos generales los reflujos de grado I-III se tratan habitualmente con antibioterapia profiláctica al menos durante un año, en espera de que culmine el proceso de desarrollo del mecanismo valvular que supone la unión ureterovesical, siempre que no aparezcan infecciones que puedan producir un deterioro renal. Cuando esto sucede o el reflujo es de grado IV-V de la clasificación internacional de enfermedades (CIE) está indicado el tratamiento quirúrgico.

Con las diversas técnicas de cirugía abierta se logra la curación de un porcentaje cercano al 90\% de los casos $^{1}$ con un coste económico elevado debido a los gastos de quirófano y a la hospitalización que requiere el postoperatorio. La aparición de la técnica endoscópica con inyección subureteral de politetrafluoroetileno $\left(\right.$ Teflon $\left.^{\circledR}\right)$, descrita por Matouschek ${ }^{3}$ y desarrollada inicialmente por O`Donnell y Puri $^{4}$ (a la que denominaron STING) abrió el campo a una terapia que, aunque proporciona unas tasas de curación menores, tiene un coste y una morbimortalidad inferiores.

Son pocas las publicaciones que analizan sus resultados en RVU asociados a patologías como vejiga neurógena, sistemas dobles, ureterocele, valvas uretrales posteriores, extrofias vesicales o reimplantación ureteral previa por estenosis ureterovesical o por reflujo recidivado tras tratamiento quirúrgico. En estas situaciones el reflujo se denomina "complicado o secundario". El objetivo de nuestro trabajo es la revisión en nuestra serie de la proporción de RVU complicados tratados endoscópicamente, determinando el porcentaje de tratamientos endoscópicos satisfactorios (aquellos con reflujo post-tratamiento con grado igual o menor a I de la Clasificación Internacional de Enfermedades, CIE) y su relación con el grado del RVU.

\section{MATERIAL Y MÉTODOS}

Revisamos los casos de reflujo vesical secundario (vejiga neurógena, doble sistemas, valvas uretrales posteriores, ureterocele, recidivado tras reimplante ureterovesical por reflujo o por estenosis previa) tratados en nuestro servicio desde la implantación en éste del tratamiento endoscópico del reflujo en 1992 hasta 2003.

La intervención se realiza mediante la utilización de un cistoscopio infantil CIRCOM ACMY del 9,4 Ch., en pacientes bajo anestesia general por tratarse de niños. Tras localizarse cistoscópicamente el meato ureteral que refluye se realiza una punción en la submucosa de la zona meatal inferior, en la teórica posición horaria de las 6, con una aguja del 4 Ch., inyectándose la sustancia que empleemos, hasta conseguir un habón que ocluya suficientemente el meato, según la técnica descrita por Puri y O'Donnell ${ }^{5}$. No es necesario el sondaje vesical posterior.

Realizamos un control ecográfico en las primeras $24 \mathrm{~h}$ postintervención con el fin de asegurar un correcto drenaje ureteral, dándosele el alta hospitalaria al paciente entonces.

El control ambulatorio del paciente lo hemos realizado con cistografía miccional seriada (CUMS) o cistograma isotópico a los 3 y 9 meses. Ante la desaparición del reflujo, y si el resto de patologias urológicas lo permiten dado que se tratan de reflujos complejos, el paciente es dado de alta. Si el reflujo persiste en grados II- III a los 9 meses de la intervención repetimos el tratamiento endoscópico, incluso hasta un tercer intento. Si el reflujo es de mayor grado o la patología de base así lo aconseja preferimos pasar a reimplantación abierta, habitualmente mediante técnica de Cohen. Consideramos que el tratamiento ha tenido éxito cuando el reflujo se hace de grado I o desaparece a los 9 meses.

La profilaxis antibiótica se realiza fundamentalmente con amoxicilina-clavulánico a dosis ajustadas según peso, comenzando la noche previa a la intervención y manteniéndola durante tres días. Posteriormente el paciente continúa con dosis profiláctica nocturna hasta la próxima revisión. 
Inicialmente (entre los años 92 y 98) empleamos el politetrafluoroetileno $\left(\right.$ Teflon $\left.^{\circledR}\right)$ en 57 pacientes (suponiendo 67 unidades excretoras), comenzando a usar el polidimetilsiloxane (Macroplastique $^{\circledR}$ ) desde 1997 (158 niños, 164 unidades excretoras) y el dextranomero-copolímero de ácido hialurónico $\left(\right.$ Deflux $\left.^{\circledR}\right)$ (50, 61 unidades excretoras) desde 2000 .

De todos estos pacientes $74(27,92 \%)$ tenían RVU secundario, suponiendo $80(27,40 \%)$ unidades refluyentes. La distribución según el material empleado fue 31 niños tratados con macroplastique (33 unidades refluyentes), 10 con deflux (11 unidades refluyentes) y 33 con teflon (36 unidades refluyentes). Tenían una edad media de 5.31 años (rango 1-12), y una distribución por sexos de 41 varones $(56,91 \%)$ y 33 hembras.

La etiología del reflujo secundario fue (Fig. 1):

- Vejigas neurógenas: 24 casos, 25 unidades refluyentes.

- Dobles sistemas: 20 casos, 23 unidades refluyentes.

- Reimplante por

- Estenosis: 6.

- Reflujo vesicoureteral primario: 13

(14 unidades)

- Valvas uretrales posteriores: 5 casos, 6 unidades refluyentes

- Extrofia vesical: 1 caso.

- Ureterocele: 5 casos.

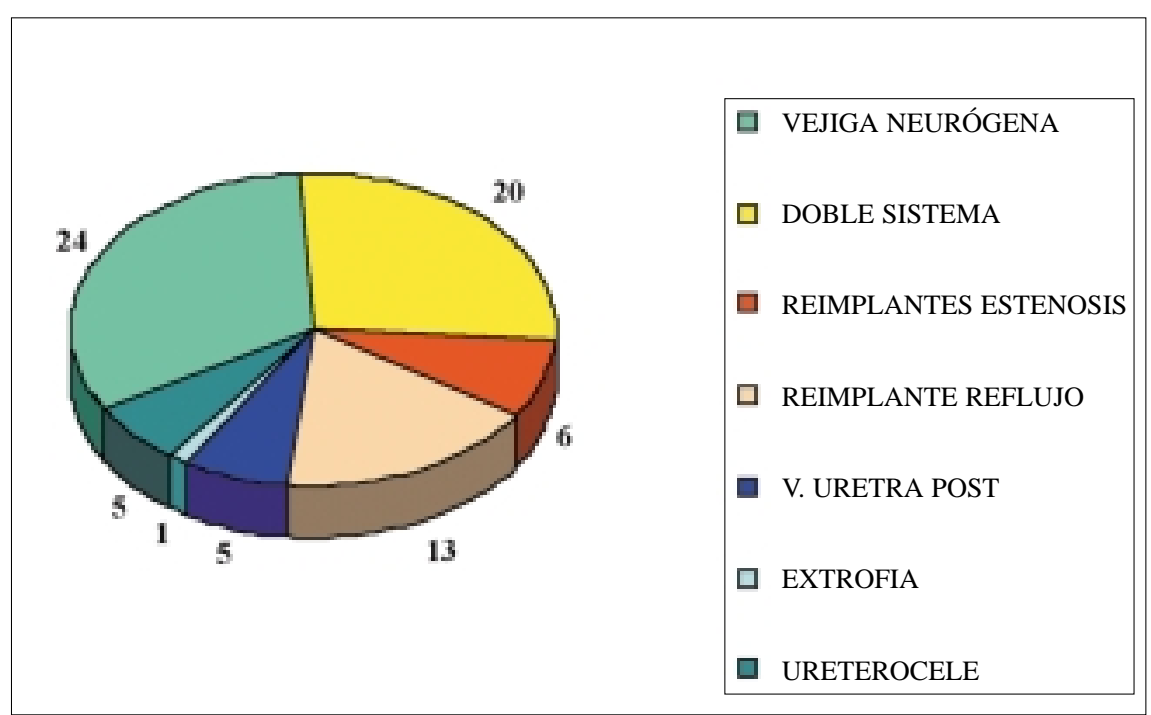

FIGURA 1. Etiologia del reflujo secundario.
Los grados de reflujo fueron $66(82,5 \%)$ unidades refluyentes con grado 3 , nueve con grado 2 $(11,25 \%)$ y $5(6,25 \%)$ con grado 4 (Figura 2 ).

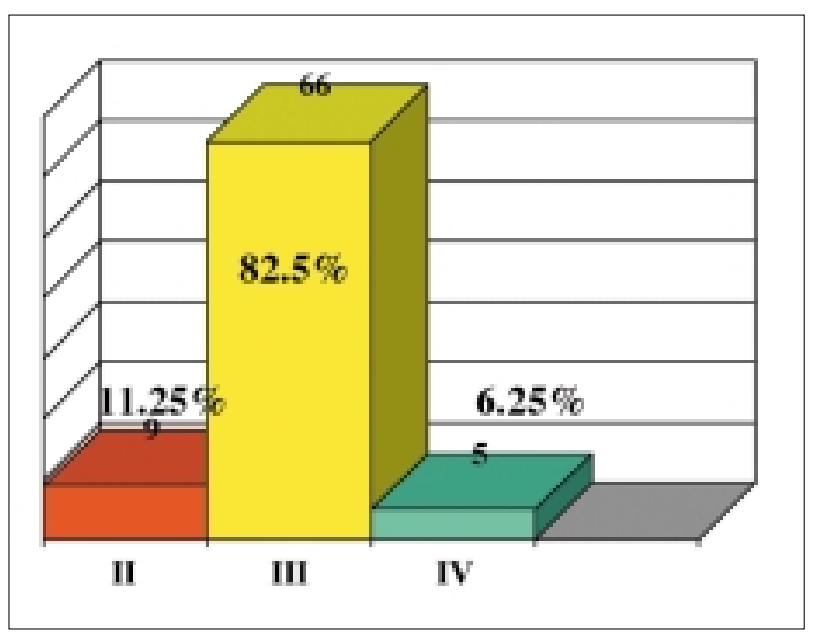

FIGURA 2. Distribución por grados de los reflujos.

\section{RESULTADOS}

Los resultados obtenidos tras la realización de las sucesivas inyecciones con los distintos materiales, con un volumen medio de 0,55 cc (rango 0,1-0,9) están resumidos en la Tabla 1.

En $69(86,25 \%)$ de los sistemas sometidos a inyección periureteral no fue preciso otro tratamiento quirúrgico (en 1 caso con grado III ante la mala evolución y las características del paciente se decidió realizar un reimplante tipo Glenn). Un segundo intento solucionó 6 de 10 reflujos, y un tercero 2 de los 4 uréteres restantes, acumulando un éxito final del 96,25\%.

Los porcentajes de éxito tras un intento oscilan entre $80 \%$ en los 5 ureteroceles hasta un $100 \%$ en el único caso de extrofia vesical y en los 6 con válvulas de uretra posterior, pasando por $86,96 \%$ en los 23 sistemas dobles, $84 \%$ en 25 vejigas neurógenas, $85,71 \%$ en los 14 uréteres reimplantados por reflujo primario, y $86,33 \%$ en los 6 uréteres reimplantados por estenosis de la unión ureterovesical (Tabla 1). 
Tabla 1

Porcentaje de éxito de las punciones según etiología del reflujo y material

\begin{tabular}{|c|c|c|c|c|c|}
\hline $\begin{array}{l}\text { UNIDADES REFLUYENTES } \\
\text { SEGÚN PATOLOGÍA ASOCIADA } \\
\text { Y MATERIAL }\end{array}$ & $\underset{n}{\text { TEFLON }}$ & $\begin{array}{c}\text { MACROPL } \\
\mathbf{n}\end{array}$ & $\underset{\mathbf{n}}{\operatorname{DEFLUX}}$ & Total & $\begin{array}{l}\text { Intentos realizados } \\
\text { (\% éxito en 19) }\end{array}$ \\
\hline Dobles sistemas & 3 & 14 & 6 & 23 & $\begin{array}{l}1^{\circ}: 21^{*}(86,96 \%) \\
2^{\circ}: \text { uno } \\
3^{\circ}: \text { uno }\end{array}$ \\
\hline Vejigas neurógenas & & 13 & 9 & 3 & $\begin{array}{l}1^{\circ}: 21(84 \%) \\
252^{\circ}: \text { tres } \\
3^{\text {oo: uno }}\end{array}$ \\
\hline Válvulas uretra posterior & 4 & 1 & 1 & 6 & $\begin{array}{l}1^{\circ}: 6(100 \%) \\
2^{\circ}: \text { ninguno } \\
3^{\circ}: \text { ninguno }\end{array}$ \\
\hline Ureteroceles & 4 & 1 & - & 5 & $\begin{array}{l}1^{\circ}: 4(80 \%) \\
2^{\text {o: }} \text { ninguno } \\
3^{\text {o: uno }}\end{array}$ \\
\hline Estenosis. Reimplantación & 3 & 3 & & 6 & $\begin{array}{l}1^{\circ}: 5(86.33 \%) \\
2^{\circ}: \text { ninguno } \\
3^{\circ}: \text { uno }\end{array}$ \\
\hline RVU primario. Reimplante & 8 & 5 & 1 & 14 & $\begin{array}{l}1^{\circ}: 12(85.71 \%) \\
2^{\circ}: \text { dos } \\
3^{\text {o: }} \text { ninguno }\end{array}$ \\
\hline \multirow[t]{2}{*}{ Extrofia vesical } & 1 & - & - & 1 & $\begin{array}{l}1^{\circ}: 1(100 \%) \\
2^{\circ}: \text { ninguno } \\
3^{\circ}: \text { ninguno }\end{array}$ \\
\hline & 36 & 33 & 11 & 80 & $\begin{array}{l}1^{\circ}: 70^{*}(86.25 \%) \\
2^{\circ}: 6 \\
3^{\circ}: 4\end{array}$ \\
\hline
\end{tabular}

*En un caso, tras un primer tratamiento endoscópico, se realizó una reimplantación.

El porcentaje de éxito tras un intento según el grado de reflujo se distribuyó de las siguiente forma: $100 \%$ en los de grado IV, $83,33 \%$ en los de grado III, $88,9 \%$ en los de grado II.

En la Tabla 2 se detallan las particularidades de los casos en que fue necesario más de un intento para conseguir la corrección del reflujo.

\section{DISCUSIÓN}

El RVU es la patología urológica mas frecuentemente asociada con la infección urinaria en niños. Dependiendo del grado del reflujo, entre el 30-70\% de los pacientes presentará lesiones renales al diagnóstico ${ }^{6}$. La tendencia es a la resolución espontánea, aunque en otros pacientes puede conducir a una nefropatía por reflujo, cuya patogénesis es básicamente infecciosa. Estos aspectos han hecho que la idoneidad de los distintos tratamientos empleados esté en continua discusión.

La aparición en los últimos años de las técnicas endoscópicas para el tratamiento del RVU ha determinado en nuestro país una gran variación en la práctica terapéutica. Parece haber una opinión generalizada sobre que las indicaciones de cirugía abierta debería ser restringida a grados altos con elevado riesgo o acompañados por otras patologías urológicas severas, dado que en general los procedimientos endoscópicos son más cortos, con menos coste y más fácilmente realizables, incluso de manera ambulatoria ${ }^{7}$. Además el tratamiento endoscópico ha producido un descenso en la indicación de tratamientos médicos prolongados, al considerarse menos agresivo que éste ${ }^{8}$.

La efectividad de la terapia endoscópica depende fundamentalmente del grado del reflujo ${ }^{6}$, siendo mayor cuanto menor sea el grado. En nuestra serie sin embargo el mejor resultado lo encontramos en los pacientes con grado IV. Esto es fácilmente explicable viendo la desproporción en los grupos según el grado de reflujo (69\% de unidades eran grado III, mientras que sólo un 6,25\% eran grado IV), indicativa de la selección cuidadosa a la que han sido sometidos los pacientes con grado IV antes de ser 
Tabla 2

Descripción de pacientes con más de un intento

\begin{tabular}{|c|c|c|c|c|c|c|}
\hline $\begin{array}{l}\text { Malformación/ } \\
\text { factor previo }\end{array}$ & $\begin{array}{l}\text { Evolució } \\
\text { Pre-tto } \\
\text { n }\end{array}$ & $\begin{array}{l}\text { n Grado } \\
\text { Post-tto } \\
\text { n }\end{array}$ & $\begin{array}{c}\text { Cirugía previa } \\
\text { al Sting }\end{array}$ & $\begin{array}{c}\text { Intentos } \\
\mathbf{n}\end{array}$ & Material & $\begin{array}{l}\text { Edad } \\
\mathbf{n}\end{array}$ \\
\hline Doble sistema & 3 & 3 & $\begin{array}{l}\text { Vesicostomía. } \\
\text { Ureterostomia } \\
\text { Politano }\end{array}$ & 3 & MACROPL & 6 \\
\hline Vejiga neurógena & 3 & 3 & & 3 & MACROPL & 5 \\
\hline Estenosis U-V & 3 & 3 & $\begin{array}{l}\text { Vesicostomía y } \\
\text { meatotomía bilateral } \\
\text { Ureterostomía bilateral }\end{array}$ & 1 & MACROPL & 1 \\
\hline $\begin{array}{l}\text { Hipoplasia vesical } \\
\text { Reflujo }\end{array}$ & 2 & 3 & Plastia vesical. Glenn & 2 & MACROPL & 1 \\
\hline Reflujo primario & 3 & 3 & Glenn bilateral & 2 & MACROPL & 5 \\
\hline Vejiga neurógena & 3 & 3 & & 2 & DEFLUX & 1 \\
\hline Doble sistema & 3 & 3 & $\begin{array}{c}\text { Ureterocele. Ureterohe } \\
\text { minefrectomía } \\
\text { HRSI* }^{*}\end{array}$ & 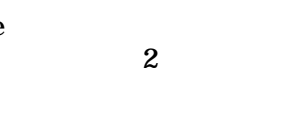 & DEFLUX & 2 \\
\hline Doble sistema & 3 & 3 & & $\begin{array}{l}\text { Se hizo un Glenn } \\
\text { tras un fracaso sting }\end{array}$ & DEFLUX & 5 \\
\hline Vejiga neurógena & 3 & 3 & & 2 & TEFLON & 4 \\
\hline Vejiga neurógena & 3 & 3 & & 2 & TEFLON & 10 \\
\hline Ureterocele & 3 & 3 & $\begin{array}{c}\text { Reimplante ureteral } \\
\text { Cohen }\end{array}$ & 3 & TEFLON & 5 \\
\hline
\end{tabular}

*Hemi-riñón superior izquierdo

tratados endoscópicamente, a diferencia de los que tenian grado III.

Otro factor que influye en el resultado es la presencia de "reflujo complicado o secundario". En el análisis que Morán Penco realiza sobre el tratamiento endoscópico del RVU en nuestro país 8 se estima que este tipo de reflujo supone un 13\% de los RVU tratados endoscópicamente, cifra notablemente inferior a nuestro $27,92 \%$. Esta diferencia es atribuible a que en nuestro estudio incluimos los pacientes ya reimplantados a los que hemos tratado posteriormente de manera endoscópica. Al ser un término que incluye diversas patologías asociadas resulta difícil estimar el índice de éxitos que el tratamiento endoscópico tiene en estos casos, variando desde un $61,7 \%$ en vejigas neurógenas ${ }^{9}$ hasta un $100 \%$ en casos de válvulas de uretra posterior ${ }^{10}$. Sí parece claro que, al igual que en casos de reflujo simple, el tratamiento endoscópico obtiene peores resultados que la cirugía abierta. Concretamente Aboutaleb ob- tiene un $81,8 \%$ de éxitos con tratamiento endoscópico frente a un $97,7 \%$ con reimplantación abierta en casos de RVU asociado a doble siste$\mathrm{ma}^{11}$. Frente a esto la mínima tasa de complicaciones que presenta el tratamiento endoscópico $(1,2 \%)$ hacen de éste un tratamiento altamente útil ${ }^{8}$.

Hacemos ahora una breve reseña de distintos aspectos en relación con las distintas patologías asociadas al RVU secundario.

\section{Sistemas dobles}

El RVU es la anormalidad más frecuentemente asociada a los sistemas excretores dobles completos $^{12}$. Parece además que la posibilidad de regresión espontánea en reflujos con esta característica anatómica es menor debido a la anormalidad del túnel submucoso que supone ${ }^{13}$.

La eficacia del tratamiento endoscópico en estos pacientes es inferior a la presentada por los RVU no complejos. Steinbreher concluye que el 
éxito del tratamiento con Teflon ${ }^{\circledR}$ en sistemas normales alcanza un $80 \%$ con una sola inyección mientras que en los sistemas dobles sólo se alcanzó un $57 \%$ con el primer intento, que subió a un $68 \%$ tras la segunda intervención ${ }^{14}$. Estos resultados coinciden con los encontrados anteriormente por Miyakita, cifrados en un 59\% tras el primer intento ${ }^{15}$.

Existen también diferencias en la efectividad presentada por la cirugía abierta y la endoscópica. Aboutaleb comunica sus resultados específicamente referidos a sistemas dobles, en los que alcanza un éxito tras un primer intento en el $68 \%$ de los uréteres, incrementándolo hasta el $82 \%$ cuando realiza un segundo implante de Macroplastique $\AA_{\text {, }}$ comparándolo además con el éxito inicial de la cirugía abierta (98\%), aunque sin encontrar complicaciones, y con tiempos quirúrgicos y anestésicos mucho menores ${ }^{11}$. Los resultados obtenidos por este autor son más concordantes con los presentados en nuestra experiencia, con un éxito inicial del 86,96\%.

Reunanen, usando colágeno, obtuvo una eficacia inicial del $46 \%$, que descendió hasta el $22 \%$ dos años después ${ }^{16}$.

\section{Vejiga neurógena}

La incidencia del reflujo vesicoureteral al nacimiento en pacientes con mielomeningocele va del 0 al 24\%, y si su manejo no es correcto este porcentaje puede alcanzar el $60 \%$. Si se dejan sin tratar, dos tercios de estos casos terminarán sufriendo deterioro del tracto urinario superior. La mayoría de casos pueden tratarse con cateterismo intermitente y anticolinérgicos, lo que lleva a la resolución espontánea o al descenso en el grado del reflujo en un $48-63 \%$ de los pacientes. $\mathrm{Si}$ esto no sucede el Sting es un procedimiento útil, que para algunos es de primera elección ${ }^{17}$ al presentar unos porcentajes de éxito específico en vejigas neurógenas que van desde $53,6-76,8 \%$ en una primera inyección, alcanzando un $82-86,7 \%$ de éxito acumulado tras 2-3 intentos ${ }^{18,19}$. En nuestra serie obtuvimos un resultado favorable en el $84 \%$ de los pacientes con un intento.

En algunos esta estrategia falla y es necesario un reimplante ureteral, que puede ser difícil en presencia de vejigas con mucha trabeculación. La causa del fallo del primer tratamiento endoscópi- co puede estribar en la desaparición o el desplazamiento del habón debido a la presencia de altas presiones intravesicales ${ }^{20}$, más que en el mal emplazamiento inicial, aunque nuestra experiencia es que las trabéculas dificultan la correcta localización del lugar idóneo de la punción, y esto puede ser una causa de fracaso del tratamiento. Sin embargo parece que si la disfunción miccional se trata inicialmente mediante cateterismo intermitente limpio y anticolinérgicos las posibilidades de éxito del tratamiento endoscópico aumentan $^{21}$.

Aunque la vejiga neurógena disminuye los porcentajes de éxito del tratamiento tanto endoscópico como abierto, no se han identificado patrones urodinámicos específicos predictores del éxito del tratamiento. No se ha observado una relación inversa entre presión vesical de escape y vejiga hiperrefléxica y las tasas de eficacia, como parece que debería esperarse de una forma intuitiva. Sin embargo pacientes con vejiga hiperrefléxica tienen más posibilidades de tener las complicaciones infecciosas propias del reflujo al tener presiones intravesicales altas, lanzando las bacterias al tracto urinario superior ${ }^{22,23}$. Por tanto además de la adecuada profilaxis antibiótica es importante el tratamiento correcto con anticolinérgicos para prevenir las secuelas, puesto que si se maneja bien la alta presión vesical no hay tendencia al deterioro del riñón. En todos nuestros casos la hiperreflexia fue controlada con tratamiento anticolinérgico previamente a la realización del tratamiento endoscópico. Quizás sea éste un factor determinante del alto porcentaje de éxito en el primer intento en nuestros casos (84\%).

A la hora de la indicación la impresión clínica es importante en esta compleja población de pacientes, recomendándose que uréteres con reflujo severo sean tratados con cirugía abierta en primera instancia ${ }^{9}$.

Es importante destacar que algunos artículos muestran que en el seguimiento a largo plazo los resultados no parecen ser tan óptimos. Haferdkamp publica en su serie con colágeno bovino que a los 2 años sólo el 15\% continuaban sin reflujo ${ }^{18}$. Queda por ver si nuevos materiales mejoran estos resultados a largo plazo. 


\section{Fracaso de reimplante abierto}

Aubert mantiene que las malas condiciones de la mucosa no son un problema para la técnica, recomendando que pasen al menos seis meses desde el reimplante ${ }^{21}$. Para los uréteres reimplantados mediante técnica de Cohen la inyección debe ponerse en perpendicular al túnel ureteral, distal al orificio en $2-3 \mathrm{~mm}$, de forma que se verá la subida de la mucosa posterior, pero es imposible cateterizar el uréter. Esto no influye en el uréter contralateral que cruza la línea media. Si se usa técnica de Gil-Vernet cada uréter se inyecta de manera separada.

\section{Ureterocele}

En nuestra experiencia sólo lo hemos usado asociado al reflujo de la unidad inferior tras haber realizado la ureteroheminefrectomía polar superior por anulación funcional. Asociado a esta patología el tratamiento endoscópico ha tenido el menor porcentaje en resolución del reflujo. La debilidad del trígono y la dificultad para ubicar el sitio de la inyección son factores condicionantes.

\section{Extrofia vesical}

Tras el cierre quirúrgico vesical el reflujo es prácticamente la norma, lo que en caso de alcanzar la continencia puede crear una situación peligrosa para los riñones. En este caso el tratamiento endoscópico es ideal, aunque la técnica puede verse dificultada porque los orificios se encuentran en situación posterior, con difícil acceso para la aguja ${ }^{21}$.

\section{Válvulas uretrales posteriores}

Aunque un $50-72 \%$ de los niños con válvulas uretrales posteriores presentan reflujo cuando éstas se diagnostican, son muy escasos los artículos que tratan específicamente los resultados del tratamiento endoscópico del RVU en pacientes con esta patología del tracto urinario inferior. Este reflujo es a veces primario (debido a anomalías del brote ureteral) y en otras ocasiones es secundario a hiperpresión vesical, divertículos paraureterales y pérdida de competencia valvular urétero-vesical. En aproximadamente un tercio de los casos el tratamiento de las válvulas producirá la resolución del RVU. En otro tercio éste se mantiene pero una adecuada profilaxis antibiótica será suficiente para preservar la función renal hasta la desaparición espontánea del reflujo, por lo que tras corregir las válvulas será necesario tomarse un cierto tiempo antes de tomar una actitud quirúrgica ${ }^{24}$.

Puri publica una serie de 15 pacientes (24 uréteres refluyentes) con válvulas uretrales posteriores que fueron tratados con inyección periureteral endoscópica de politetrafluoroetileno $\left(\text { Teflon }^{\circledR}\right)^{10}$. En 17 de los uréteres refluyentes el tratamiento fue exitoso con un único intento, cinco uréteres requirieron dos y dos precisaron tres, desapareciendo finalmente el RVU en las 24 unidades refluyentes, no reapareciendo en un seguimiento de un año. No se encontraron efectos adversos. En nuestros pacientes el resultado final ha sido similar, consiguiéndose la resolución del reflujo en el $100 \%$ de casos.

En nuestra serie se han encontrado 5 pacientes (6 unidades refluyentes, todas grado III excepto una grado IV) tratados endoscópicamente de RVU asociado a válvulas de uretra posterior. En tres de ellos se había realizado una reimplantación ureteral previa (todas tipo Cohen) cuyo resultado no fue satisfactorio. En uno de los pacientes restantes el RVU se asociaba además a un ureterocele, que fue tratado de manera simultánea a las válvulas uretrales de manera endoscópica. En todos los casos tras una inyección periureteral (que fue de politetrafluoroetileno en tres de ellos, de dextranómerocopolímero de ácido hialurónico en una, y de polidimetilsiloxane en otra) el reflujo desapareció.

\section{CONCLUSIONES}

En nuestra serie el tratamiento endoscópico del RVU ha resultado de utilidad incluso en casos complejos, con diversas patologías asociadas, por lo que creemos que debe considerarse como el tratamiento de elección de forma inicial.

Una correcta selección de los pacientes subsidiarios de este tipo de tratamiento, basándonos en la valoración de factores como extravesicalización de la unión ureterovesical o la presencia de divertículos paraureterales, nos permitirá obtener unos resultados satisfactorios.

Pacientes en los que confluyen varios factores deben ser seleccionados individualmente para obtener resultados favorables. 


\section{REFERENCIAS}

1. López Lópeza JA, Sánchez Zalabardo JM, Sánchez Elipe MA, Valdivia Uría JG, Valle Gerholda J, Jiménez Vidal A: Tratamiento endoscópico del reflujo vesicoureteral. An Esp Pediatr 2001;54:132-135.

2. Gosalvez R: Reflujo vesicoureteral. En: Garat JM, Gosalvez R Eds. Urología Pediátrica. Ed. Salvat 1987; Cap.11:271287.

3. Matouschek E: Sobre un nuevo concepto para el tratamiento del reflujo vesicoureteral. Arch Esp Urol 1981;34: 384-385.

4. O`Donnell B, Puri P: Treatment of vesicoureteric reflux by endoscopic injection of Teflon. Brit Med J 1984;289:7-9.

5. O`Donnel B, Puri P. Endoscopic correction of primary vesicuretic reflux: results in 94 ureters. BMJ 1986;293:14041406.

6. Vela D, Montero M, Méndez R, Gómez Tellado M, País E: Contraindicaciones relativas para el tratamiento endoscópico del reflujo vesicoureteral. Cir Pediatr 2000;13:141144 .

7. Kershen RT, Atala A: New advances in injectable therapies for the treatment of incontinence and vesicoureteral reflux. Urol Clin North Am 1999;26:81-94.

8. Moran Penco JM, Gómez Fraile A, Rodríguez Alarcón J, García Merino F, Vela D, Sanjuan Rodriguez S, Aransay Brantot A: Evolution of the treatment of vesicoureteral reflux in Spain. J Urol 2004;171:834-837.

9. Engel JD, Palmer LS, Cheng EY, Kaplan WE: Surgical versus endoscopic correction of vesicoureteral reflux in children with neurogenic bladder dysfunction. J Urol 1997;157(6):2291-2294.

10. Puri P, Kumar R: Endoscopio correction of vesicoureteral reflux secondary to posterior urethral valves. J Urol 1996;156(2S):680-682.

11. Aboutaleb H, Bolduc S, Khoury AE, Upadhyay J, Bagli DJ, Farhat W: Polydimethylsiloxane injection versus open surgery for the treatment of vesicoureteral reflux in complete duplex systems. J Urol 2003;170:1563-1565.

12. Lee PH, Diamond DA, Duffy PG, Ransley PG: Duplex reflux: a study of 105 children. J Urol 1991;146:657-691.

13. Caldamone AA: Duplication anomalies of the upper tract in infants and children. Urol Clin North Am 1985;12:7591
14. Steinbrecher HA, Edwards B, Malone PSJ: The STING in refluxing duplex system. British J Urol 1995; 76:165-168.

15. Miyakitah, Ninan GK, Puri P: Endoscopic correction of vesicoureteric reflux in duplex systems. Eur J Urol 1993; 24:111-1115.

16. Reunanen M: Endoscopic collagen injection: its limits in correcting vesico-ureteral reflux in duplicated ureters. Eur Urol 1997;31:243-245.

17. Misra D: Efficacy of STING in the treatment of vesicoureteric reflux in neuropathic bladder. Br J Urol 1996; 77:328-329.

18. Haferkamp A, Möhring K, Staehler G, Gerner HJ, Dörsam $\mathrm{J}$ :Long-term efficacy of subureteral collagen injection for endoscopic treatment of vesicoureteral reflux in neurogenic bladder cases. J Urol 2000;163:274-277.

19. Misra, D, Potts, SR, Brown, S et al: Endoscopic treatment of vesico-ureteric reflux in neurogenic bladder-8 years experience. J Pediatr Surg 1996;31:1262-1264.

20. Capozza N, Lais A, Matarazzo E, Nappo S, Patricolo M, Caione P: influence of voiding dysfunction on the outcome of endoscopic treatment for vesicoureteral reflux. J Urol 2002;168:1695-1698.

21. Aubert D, Zoupanos G, Destuynder O, Hurez F: “Sting” procedure in the treatment of secondary reflux in children. Eur Urol 1990;17:307-309.

22. Naseer SR, Steinhardt GF: New renal scars in children with urinary tract infections, vesicoureteral reflux and voiding dysfunction: a prospective evaluation. J Urol 1997; 158:566-568.

23. Greenfield SP, Wan J: The relationship between dysfunction voiding and congenital vesicoureteral reflux. Curr Opin Urol 2000;10:607-610.

24. Gonzales ET: Válvulas uretrales posteriores y otras anomalías de la uretra. En Walsh PC, Retik AB, Stamey TA, Darracott Eds. Campbell Urología. Ed. Panamericana SA 1994; Cap 49:1854-1873.

D. Enrique Argüelles Salido

Milano Plomizo, 4 - portal 4 - 1ㅇ B

41020 Sevilla

E-mail: earguelles@telefonica.net

(Trabajo recibido el 11 noviembre de 2004) 